\title{
Adiaspiromicose pulmonar humana
}

Human pulmonary adiaspiromycosis

Lina Gomes dos Santos'; José Klérton Luz Araújo²; Ana Carolina Brito Tavares²; João dos Martírios de Araújo Moura Fé3

unitermos

Emmonsia crescens

Adiaspiromicose

Conídios

Biópsia pulmonar cirúrgica

Patologia

\section{resumo}

A adiaspiromicose é uma doença fúngica sistêmica que acomete usualmente roedores e raramente atinge o homem. É causada pelo fungo Emmonsia crescens e ocorre após a inalação da forma contagiante (conídios). Embora estas formas não se multipliquem nem se disseminem no organismo humano, induzem uma reação inflamatória crônica granulomatosa de padrão miliar que pode levar a falência respiratória e morte. Apresentamos aqui um caso de adiaspiromicose pulmonar humana em paciente imunocompetente que exibia infiltrado intersticial pulmonar difuso ao exame de imagem e fora diagnosticado mediante biópsia pulmonar.

\section{abstract}

Adiaspiromycosis is a systemic fungal disease that usually affects rodents and rarely infects humans. It is caused by the fungus Emmonsia crescens and occurs after inhalation of its contagious form (conidia). Although these forms neither multiply nor spread in the human body, they cause a chronic granulomatous inflammatory reaction of miliary pattern, which may lead to respiratory failure and death. In this study we present a case of human pulmonary adiaspiromycosis in an immunocompetent patient that showed a diffuse pulmonary interstitial infiltrate diagnosed by pulmonary biopsy. key words

Emmonsia crescens

Adiaspiromycosis

Conidia

Pulmonary surgical biopsy

Pathology

1. Mestra; médica patologista; professora de Patologia da Universidade Federal do Piauí (UFPI).

3. Médico-cirurgião torácico do Hospital São Marcos, Teresina-Pl. 


\section{Introdução}

A adiaspiromicose pulmonar é uma doença fúngica sistêmica causada por Emmonsia parva var. $\operatorname{crescens}^{(3,5,7)}$, que são encontrados no solo e acometem habitualmente roedores e pequenos mamíferos silvestres ${ }^{(2,4,6,9)}$. O contágio humano ocorre pela inalação da forma contagiante do fungo, os conídios, que medem de 2 a $4 \mu \mathrm{m}$ e, assim, alcançam facilmente os alvéolos pulmonares, onde crescem atingindo entre 300 a $700 \mu \mathrm{m}$ (adiaconídios) $)^{(2,3,8,10)}$. Não se multiplicam nem disseminam nos tecidos do hospedeiro, fenômeno único entre os fungos patogênicos ${ }^{(4)}$. A intensa resposta inflamatória granulomatosa tecidual leva a degeneração e morte do agente, tornando-a uma doença autolimitada ${ }^{(1,8)}$. Apresentamos aqui um caso de adiaspiromicose pulmonar diagnosticado mediante biópsia.

\section{Relato de caso}

Paciente do sexo masculino, 55 anos, procedente do estado do Tocantins, habitante da zona rural e trabalhador na agricultura, procurou o serviço médico com queixa de dificuldade respiratória há cerca de três meses. Apresentava quadro de astenia, tosse não-produtiva, dispneia e discreta perda ponderal. Negava tabagismo ou outras doenças pulmonares anteriores. À ausculta pulmonar havia redução do murmúrio vesicular bilateral. O exame radiológico convencional (Figura 1) e uma

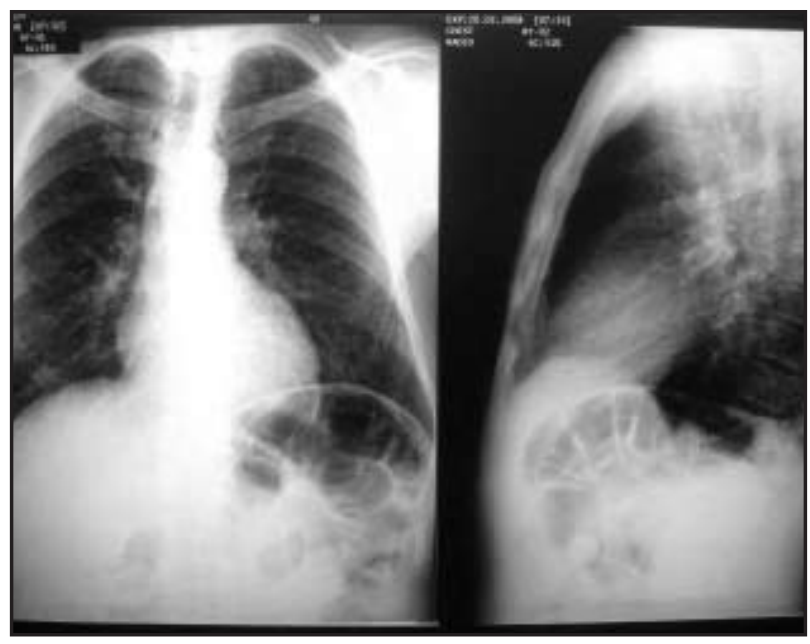

Figura 1 - Radiografia do tórax mostrando infiltrado micronodular difuso tomografia computadorizada (TC) de tórax (Figura 2) demonstraram infiltrado pulmonar micronodular difuso sem adenomegalias mediastinais ou hilares. Submetido à biópsia pulmonar incisional, o estudo histopatológico (Figuras 3 e 4) demonstrou, em coloração de rotina, processo inflamatório crônico granulomatoso padrão miliar, evidenciando granulomas centrados por esférulas de paredes espessas (adiaconídios), por vezes degeneradas, consistente com o diagnóstico de adiaspiromicose. As colorações do ácido periódico de Schiff (PAS) e de Grocott marcaram fortemente tais estruturas, confirmando o diagnóstico morfológico de adiaspiromicose pulmonar.

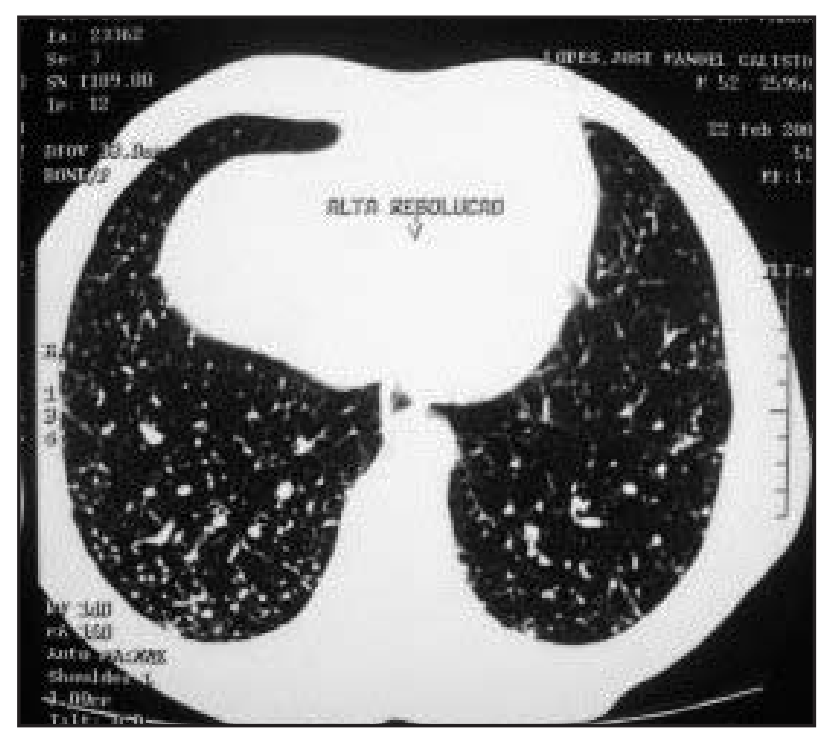

Figura 2 - Tomografia de tórax mostrando infiltrado micronodular difuso

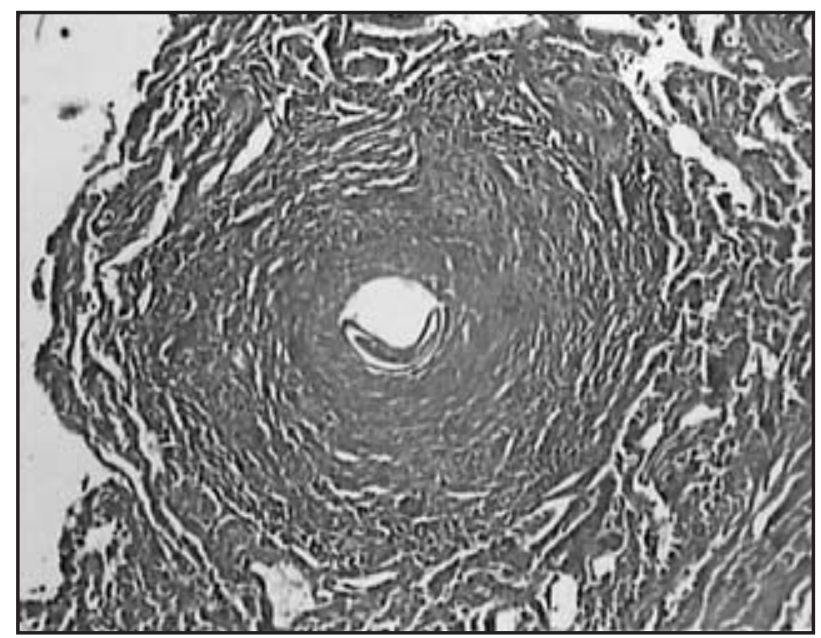

Figura 3 - Fotomicroscopia evidenciando reação granulomatosa não-necrotizante centrada por fungo (adiaconídio) no tecido pulmonar (HE, 40X) 


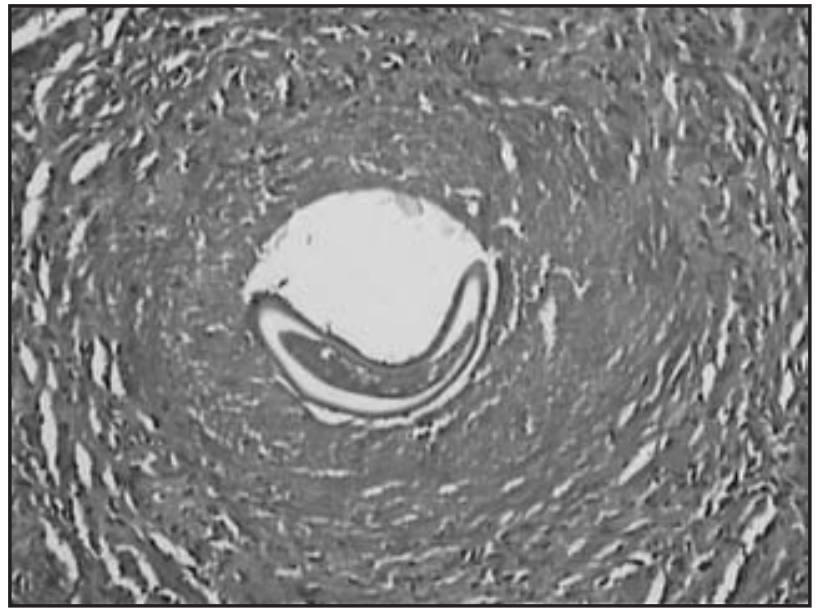

Figura 4 - Fotomicroscopia evidenciando reação granulomatosa não-necrotizante centrada por fungo (adiaconídio) no tecido pulmonar (HE, 100x)

\section{Discussão}

O primeiro caso de adiaspiromicose foi publicado na França, em 1964(1). Do nosso conhecimento, até o ano de 2008, existem 41 casos de adiaspiromicose publicados em todo o mundo, mais da metade (23 casos) oriunda do Brasil, sendo este o $24^{\circ}$ caso publicado. $O$ caso aqui descrito apresentou quadro respiratório discreto, possivelmente pela inalação de pequena quantidade da forma infectante do fungo (conídios) presente no solo. Aceita-se que esses fungos vivam saprofiticamente no solo, o que explicaria a presença de adiaconídios nos pulmões de pequenos mamíferos fossadores. Além disso, poderia explicar sua maior ocorrência em rurícolas, embora até o momento não se conheça a maneira exata como eles atingem o organismo do hospedeiro ${ }^{(4)}$.

Em 1997, Barbosa et al.(1) descreveram 19 casos de adiaspiromicose e propuseram os seguintes critérios para o diagnóstico: início agudo de sintomatologia sistêmica e respiratória, lesões micronodulares difusas e bilaterais ao exame radiológico e identificação histológica de granulomas com adiaconídios. Na presença de tais achados, propuseram a terminologia adiaspiromicose pulmonar aguda.

A intensidade da infecção está diretamente associada à quantidade de conídios inalada, às enfermidades associadas, à antigenicidade do fungo e à resposta imune do hospedeiro(3), o que ocasiona quadros subclínicos ou mesmo doença grave e fatal|(5-7, 9). Cada adiaconídio suscita uma reação inflamatória local. Ao atingir os alvéolos, crescem rapidamente, degeneram-se e morrem como consequência da própria reação tissular que induzem no hospedeiro, o que impede, até o momento, o cultivo desses fungos a partir de amostras teciduais humanas ${ }^{(5)}$. A reação inflamatória leva à formação de granulomas com fibrose concêntrica, que envolve e isola os adiaconídios nos tecidos, impedindo sua eliminação pelo escarro ou aspirado brônquico, tornando o diagnóstico difíci(1). Para tal, o diagnóstico definitivo da doença é feito mediante a identificação de adiaconídios em cortes histológicos de tecido pulmonar ${ }^{(1,4)}$.

Por se tratar de doença autolimitada, a introdução de terapêutica antifúngica deve ser criteriosa, pois ainda não há comprovação do efeito letal dessas drogas sobre os adiaconídios tissulares. No presente caso, por se tratar de quadro clínico discreto, não foi introduzido antifúngico, obtendo-se melhora do quadro apenas com sintomáticos.

\section{Referências}

1. DE ALMEIDA BARBOSA, A.; MOREIRA LEMOS A. C.; SEVERO, L. C. Acute pulmonary adiaspiromycosis. Report of three cases and review of 16 other cases collected from the literature. Rev Iberoam Micol, v. 14, n. 4, p. 177-80, 1997.

2. DOS SANTOS, V. M. et al. Adiaspiromicose pulmonar disseminada: relato de caso. Rev Soc Bras Med Trop, v. 30, n. 5, p. 397-400, 1997.

3. DOS SANTOS, V.M. et al. Contribuição ao diagnóstico morfológico da adiaspiromicose pulmonar. Rev Soc Bras Med Trop, v. 33, n. 5, p. 493-7, 2000.
4. LIMA, T. S. M. et al. Novo caso de adiaspiromicose humana diagnosticado por biópsia transbrônquica. J Pneumol, v. 24, n. 5, p. 339-41, 1998.

05. MARTINS, R. L. M. et al. Adiaspiromicose humana: relato de um caso tratado com cetoconazol. Rev Soc Bras Med Trop, v. 30, n. 6, p. 507-9, 1997.

6. MORAES, M. A. P. et al. Caso fatal de adiaspiromicose pulmonar humana. Rev Inst. Méd Trop São Paulo, v. 31, p. 118-94, 1989.

7. MORAES, M. A. P. et al. Adiaspiromicose pulmonar: achado casual em paciente falecido de febre 
amarela. Rev Soc Bras Med Trop, v. 34, n. 1, p. 83-5, 2001.

8. MORAES, M. A. P.; GOMES, M. I. Adiaspiromicose humana: lesões cicatriciais em linfonodos do mediastino. Rev Soc Bras Med Trop, v. 37, n. 2, p. 177-8, 2004.
09. PERES, L. C. et al. Fulminant disseminated pulmonary adiaspiromycosis in humans. Am J Trop Med Hyg, v. 34, n. 1, p. 83-5, 2001.

10. SUN, Y. et al. Fine needle aspiration of pulmonary adiaspiromycosis: a case report. Acta Cytol, v. 51, n. 2, p. 217-21, 2007. 